\title{
Tuberculosis pericárdica con efusión hemorrágica
}

\section{Pericardial tuberculosis with hemorrhagic effusion}

\author{
Raúl Herrera-Valdivia,' Christian Rojas-Contreras, ${ }^{2}$ \\ Eduardo De la Cruz-Yaya ${ }^{3}$ y Mery Florián-Molina ${ }^{4}$
}

Herrera-Valdivia R, Rojas-Contreras C, De la CruzYaya E, Florián-Molina M. Tuberculosis pericárdica con efusión hemorrágica. Rev Soc Peru Med Interna. 2018;32(2):66-69.

https://doi.org//0.36393/spmi.v32i2.221

\section{RESUMEN}

Se presenta el caso de un paciente varón de 19 años con tres semanas de enfermedad, fiebre, diaforesis, tos, pérdida de peso, disnea progresiva. Llegó al servicio de Emergencia con síntomas de insuficiencia cardiaca. Los estudios demostraron derrame pericárdico moderado. Se realizó una ventana pericárdica donde se evidenció liquido pericárdico hemorrágico. El estudio histológico del pericardio y de un ganglio mediastínico reveló un infiltrado crónico granulomatoso y caseificante, sugerente de tuberculosis pericárdica. Respondió favorablemente al tratamiento antituberculoso más prednisona.

Palabras clave. Tuberculosis, pericarditis, efusión pericárdica.

\section{ABSTRACT}

We present the case of a 19 year-old male patient with three weeks of illness, with diaphoresis, fever, weight loss, cough, progressive dyspnea, and arrived to emergency ward with symptoms of heart failure. Studies showed moderate pericardial effusion.

He underwent a pericardial window and a hemorrhagic pericardial liquid was found. The histological study of pericardium and mediastinal lymph node revealed a chronic granulomatous and caseous infiltrate, suggestive of pericardial tuberculosis. He responded favorably to antituberculous treatment plus prednisone.

KEY WORDS. Tuberculosis, pericarditis, pericardial effusion.

I. Departamento de Medicina Interna, Hospital Militar Central, Lima.

2. Departamento de Medicina Interna/Infectologia Hospital Militar Central, Lima.

3. Departamento de Cardiología. Hospital Militar Central, Lima.

4. Estudiante de medicina.

\section{INTRODUCCIÓN}

Aunque la tuberculosis (TB) afecta en mayor proporción a los pulmones, se ha reportado a nivel mundial que entre $10 \%$ y $20 \%$ del total de casos afectan a otros órganos (extrapulmonar), lo que aún es mayor en las personas inmunodeprimidas. ${ }^{1}$ En general, esta presentación puede corresponder a la diseminación a partir de un foco primario pulmonar, sea por contigüidad o por vía linfohemática. La afectación extrapulmonar más frecuente descrita a nivel mundial es la pleural, seguida de la ganglionar, la urogenital y la osteoarticular. Otras localizaciones son muy poco frecuentes. ${ }^{2,3}$

En el Perú, en los años 2013 y 2014, casi $18 \%$ de los casos de TB reportados fueron extrapulmonares. Del total de casos notificados como TB extrapulmonar, la localización pleural fue la más frecuente (54\%), seguida de la ganglionar $(11,1 \%)$ y de la del sistema nervioso (9\%) y otras localizaciones en menor porcentaje. ${ }^{4}$ La pericarditis tuberculosa (PTB) concurre en aproximadamente $1 \%$ a $2 \%$ de pacientes con TB pulmonar, pero existe variabilidad entre países e incluso en los desarrollados. ${ }^{5}$ 
En un estudio realizado en España, en 294 pacientes inmunocompetentes con pericarditis aguda, la PTB fue diagnosticada en 12 (4\%), de los cuales 6 (2\% del total) presentaron taponamiento cardiaco y $6(2 \%)$, pericarditis constrictiva. ${ }^{6}$

Se presenta el caso de un paciente con PTB con líquido pericárdico de aspecto hemorrágico.

\section{PRESENTACIÓN DEL CASO}

Paciente varón, de 18 años, que ingresó a Emergencia del Hospital Militar Central de Lima. Refirió que en las últimas cuatro semanas había cursado con fatiga, diaforesis nocturna, hiporexia, astenia, mareos, disnea progresiva a pequeños esfuerzos y pérdida de peso de $4 \mathrm{~kg}$. En las dos semanas previas al ingreso, se agregó fiebre y tos con expectoración escasa, y, en la semana anterior al ingreso, hubo náuseas, vómitos y dolor torácico. Ingresó a Emergencia con disnea de reposo, sensación de desvanecimiento y debilidad generalizada, que le impedía deambular. No refirió ningún antecedente de importancia.

\section{Examen físico}

Mal estado general, despierto, orientado en tiempo, espacio y persona. Presión arterial 100/60 mmHg, frecuencia cardiaca $120 / \mathrm{min}$, frecuencia respiratoria 28/min, temperatura $39^{\circ} \mathrm{C}$. Marcada palidez, no edemas. Locomotor sin alteraciones.

En el cuello se evidenció un ganglio submandibular derecho de 0,5 x 1,0 cm aproximadamente, móvil de bordes regulares y no doloroso a la palpación. Ingurgitación yugular y reflujo hepatoyugular positivos.

Tórax simétrico. Buen pasaje del murmullo vesicular en ambos hemitórax. Ruidos cardiacos rítmicos, de buena intensidad, soplo en foco tricuspídeo, II/VI. Pulsos periféricos palpables.

Abdomen plano, blando y depresible. Ruidos hidroaéreos presentes. Hígado palpable a $3 \mathrm{~cm}$ por debajo del reborde costal derecho, altura hepática mayor de $14 \mathrm{~cm}$, no doloroso a la palpación.

Genitales y sistema nervioso sin alteraciones.

\section{Exámenes auxiliares}

Hemoglobina 6 g/dL, VCM 73,7 fL, HCM 23,9 pg, CHCM 32,4 g/dL. Leucocitos 7540 (segmentados $66 \%$, abastonados $0 \%$, linfocitos $26 \%$, monocitos $5 \%$, eosinófilos 3\%); plaquetas $367000 / \mathrm{mm}^{3}$.

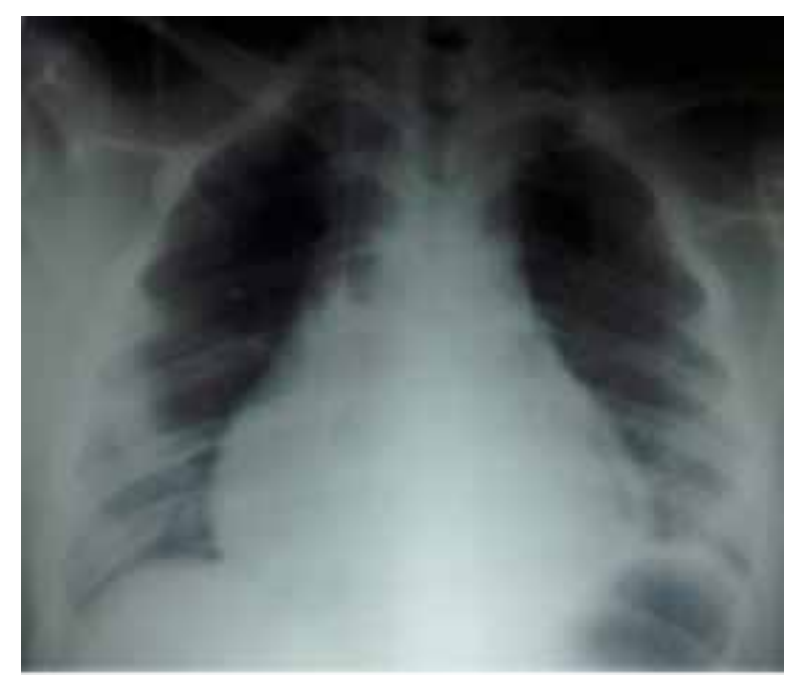

Figura I. Radiografía de tórax.

Glucosa $94 \mathrm{mg} / \mathrm{dL}$, creatinina $0,82 \mathrm{mg} / \mathrm{dL}$. Proteínas totales $7,2 \mathrm{~g} / \mathrm{dL}$, albúmina $2,7 \mathrm{mg} / \mathrm{dL}$. Bilirrubina total $0,60 \mathrm{mg} / \mathrm{dL}$. AST $58 \mathrm{UI} / \mathrm{L}$, TGP $94 \mathrm{UI} / \mathrm{L}$, fosfatasa alcalina $100 \mathrm{U} / \mathrm{L}$, lactato deshidrogenasa $430 \mathrm{U} / \mathrm{L}$.

Proteína C reactiva 171, $8 \mathrm{mg} / \mathrm{dl}$. VIH, RPR, HBsAg y anti-VHC negativos.

Examen de orina: leucocitos 1-2/campo, hematíes 0-1/ campo, proteínas trazas, nitritos y glucosa negativos.

Médula ósea normocelular, megaloblastosis mínima y disminución de las reservas de hierro.

Hemocultivo negativo. Baciloscopia de esputo seriada negativa.

Radiografía de tórax: cardiomegalia,índice cardiotorácico mayor de 0,5 , derrame pleural bilateral (Figura 1).

ECG: ritmo sinusal, frecuencia cardiaca 90 minuto, eje + 60, complejos QRS pequeños, onda $T$ negativa en V2-V3 y aplanada en V4-V6.

Ecocardiograma: derrame pericárdico moderado e imágenes ecolúcidas a nivel de pericardio, sugerente de proceso crónico (Figura 2).

Tomografía espiral multicorte de tórax: marcado engrosamiento de las hojas pericárdicas y cantidad variable de líquido pericárdico (Figura 3).

\section{Tratamiento}

Con el aumento de la disnea y ante el riesgo de taponamiento pericárdico, se le realizó una ventana pericárdica. Se halló líquido pericárdico serohemático, 

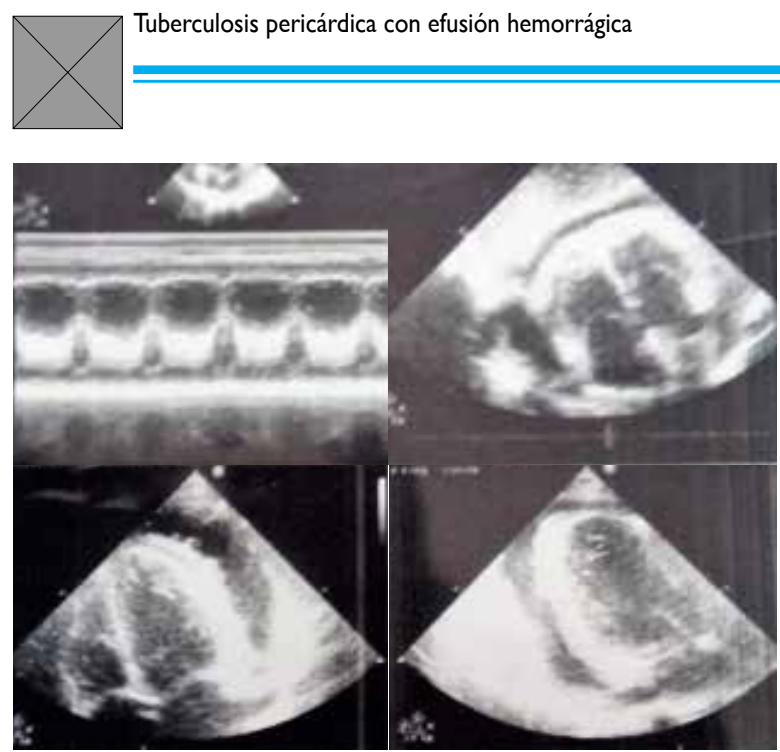

Figura 2. Ecocardiografía.

se drenó unos $100 \mathrm{~mL}$ y se le dejó un tubo de drenaje, por donde salieron $150 \mathrm{~mL}$ adicionales. El pericardio era engrosado y con adherencias. Se tomó una muestra de pericardio y un ganglio mediastínico paracardíaco para estudio histopatológico.

Con el drenaje hubo mejoría clínica, con disminución de la disnea. Al quinto día de iniciado el tratamiento, el volumen de drenaje fue cero, por lo que se retiró el tubo.

El estudio citoquímico reveló un exudado con hematíes y actividad de la adenosina desaminasa (ADA) de 64 U/L (normal: 0-25 U/L).

El estudio histológico del pericardio reveló tejido fibroso con infiltración crónica granulomatosa caseificante de probable origen tuberculoso. El estudio histológico del ganglio mediastínico mostró una linfadenitis crónica granulomatosa caseificante de probable origen tuberculoso.

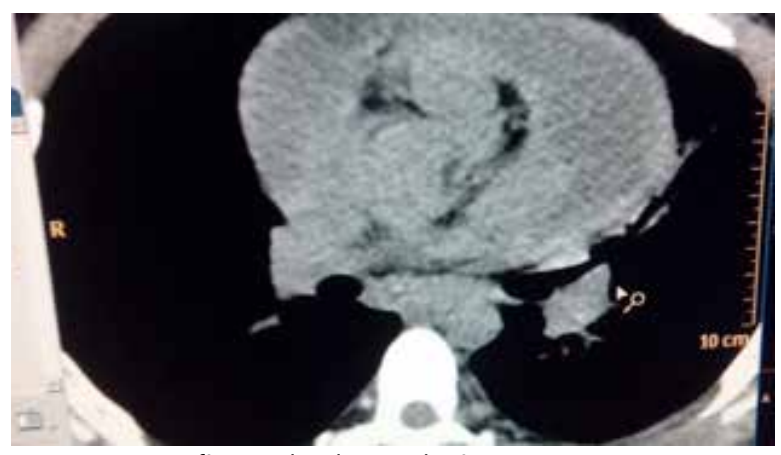

Figura 3. Tomografía espiral multicorte de tórax.

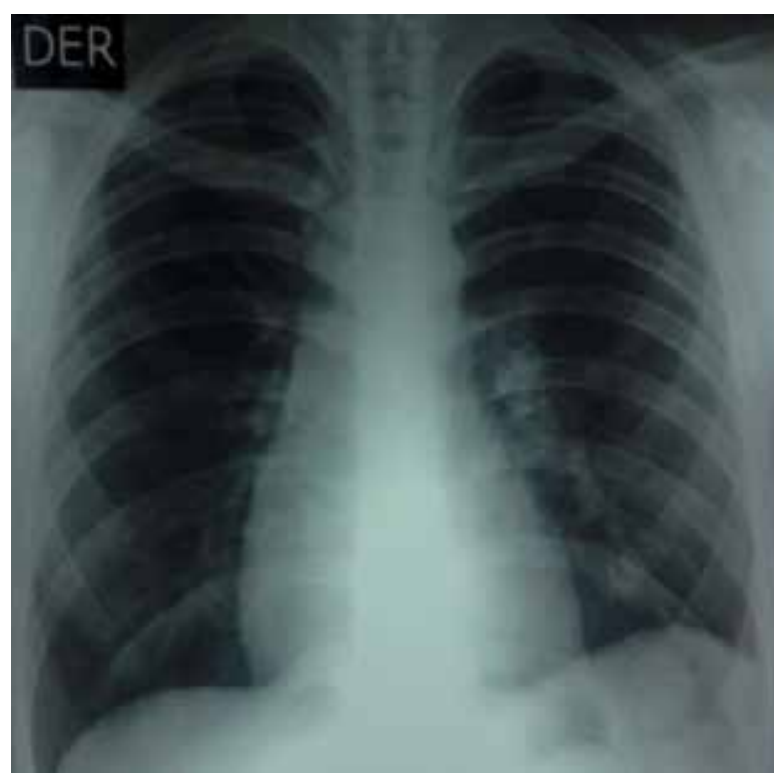

Figura 5. Radiografía de tórax a las tres semanas de tratamiento antituberculoso.

\section{Evolución}

El paciente continuó disneico y con fiebre. Recibió medidas de soporte como antibióticos de amplio espectro y transfusión de paquete globular. Por la actividad elevada de la ADA en líquido pericárdico y el engrosamiento pleural, se sospechó de TB.La tomografía del tórax no mostró adenomegalias mediastínicas, por lo que otras posibilidades como linfomas o neoplasias se alejaron. Como los hallazgos histológicos del pericardio y el ganglio fueron muy sugestivos de TB, se inició el tratamiento estándar con cuatro drogas (isoniacida, rifampicina, pirazinamida y etambutol). La respuesta favorable al tratamiento también apoya el diagnóstico. El paciente mejoró clínicamente y la fiebre disminuyó paulatinamente hasta las tres semanas de tratamiento. Esto se confirmó con los controles radiográfico (Figura 5) y ecográfico.

\section{DISCUSIÓN}

La PTB resulta de la extensión al pericardio de lesiones tuberculosas del pulmón, pleura o ganglios mediastínicos. La PTB es infrecuente y tiene una mortalidad de $80 \%$ a $90 \%$ sin tratamiento y de $14 \%$ a $40 \%$ aun con tratamiento.?

La presentación clínica de la PTB es variable, con síntomas generales como fiebre, pérdida de peso, fatiga y sudoración nocturna; síntomas cardiorrespiratorios como tos, disnea y dolor torácico. Los pacientes pueden 
presentar falla cardíaca debido a compresión cardíaca crónica; pero, también pueden presentar abruptamente taponamiento cardíaco.

Se ha descrito cuatro estadios de la PTB, seco, exudativo, absortivo o fibroso temprano y restrictivo o fibroso tardío. ${ }^{8}$ El fluido pericárdico seroso o serohemático es encontrado en el $80 \%$ de casos de PTB. ${ }^{9}$ Se reporta que la causa de pericarditis hemorrágica más común es la iatrogénica (intervención transcatéter, inserción de marcapasos). Luego, sigue el síndrome pospericardiotomía, cáncer (mama, pulmón, linfomas, etc.), complicación de infarto de miocardio, uremia, trauma, disección de aorta, anticoagulación, tuberculosis, etc. A la tuberculosis le corresponde el $2 \%$ de las efusiones pericárdicas hemorrágicas, pero en países como Perú se le debe tener presente en el diagnóstico diferencial. ${ }^{10}$

La actividad incrementada de la ADA en el líquido pericárdico tiene una sensibilidad de $89 \%$ y una especificidad de $72 \%$ para el diagnóstico de PTB. La ADA es una enzima que cataliza la desaminación de la adenosina hacia sus respectivos nucleótidos de inosina. Esta conversión es un paso inicial para una serie de reacciones responsables de la diferenciación y la proliferación de los linfocitos. Por lo tanto, la ADA es considerada como un indicador de la inmunidad mediata o celular. Los macrófagos también sintetizan ADA, lo que explica la actividad incrementada de la ADA en efusiones como la de la tuberculosis, incluidas aquellas en que el patrón de diferenciación es predominantemente de neutrófilos. La efusión pericárdica en la PTB está constituida por exudado de linfocitos, ocasionalmente neutrófilos o ambos. El análisis de la actividad de la ADA puede ser útil en el diagnóstico temprano, pues la tinción del bacilo tuberculoso en el líquido pericárdico de PTB es usualmente negativa. El cultivo mejora la sensibilidad diagnóstica a no más del $50 \%$ a expensas de mayor tiempo en el resultado. ${ }^{11}$

El diagnóstico definitivo de PTB se basa en uno de los siguientes criterios: ${ }^{12}$

- Cultivo positivo para Mycobacterium tuberculosis en tejido o líquido pericárdico.
Tinción positiva para bacilos ácido/alcoholresistentes o granulomas caseosos típicos en la biopsia pericárdica.

- Reacción en cadena de polimerasa (PCR) para tuberculosis positiva en biopsia de pericardio.

El paciente presentado cumplió con el segundo criterio además de la respuesta al tratamiento.

En conclusión, en Perú se debe considerar a la tuberculosis pericárdica como una causa importante de efusión pericárdica hemorrágica.

\section{REFERENCIAS BIBLIOGRÁFICAS}

I. Lin JN, Lai CH, Chen YH, Lee SSJ, Tsai SS, Huang CK, et al. Risk factors for extrapulmonar tuberculosis compared to pulmonary tuberculosis. Int J Tuberc Lung Dis. 2009; 13(5):620-5.

2. Garcia-Rodriguez JF, Alvarez-Diaz H, Lorenzo-Garcia MV, MarinoCallejo A, Fernandez-Rial A, Sesma-Sanchez P. Extrapulmonary tuberculosis: epidemiology and riskfactors. Enferm Infecc Microbiol Clin. 20I I Aug-Sep;29(7):502-9.

3. Peto HM, Pratt RH, Harrington TA, LoBue PA, Armstrong LR. Epidemiology of extrapulmonary tuberculosis in the United States, 1993-2006. Clin Infect Dis. 2009;49(9): 1350-7.

4. Ministerio de Salud del Perú. Dirección General de Epidemiologia. Análisis de la situación epidemiológica de la tuberculosis en el Perú, 20I5. URL disponible en: http://www.dge.gob.pe

5. Larrieu AJ, Tyers GF, Williams EH, Derrick JR. Recent experience with tuberculous pericarditis. Ann Thorac Surg. 2008;29:464.

6. Fiske CT, Griffin MR, Erin H, et al. Black race, sex, and extrapulmonary tuberculosis risk: an ob ᄀservational study. BMC Infect Dis. 2010;10:16.

7. Sosa S, López H, Padilla L. Pericardial tuberculosis: case report. Rev Med Hondur. 2010;78(I):25-28.

8. Lee VYCh, Wong JTH, Fan HCh, Yeung VFT. Tuberculous pericarditis presenting as massive haemorrhagic pericardial effusion. BMJ Case Reports. 2012.

9. Mayosi BM, Burgess LJ, Doubell AF.Tuberculous pericarditis. Circulation. 2005; I I2: 3608-3616.

10. Hayase N, Inokuchi R, Nakamura K, Horie R, Sato H, Asada T, et al. sudden cardiac arrest caused by tuberculous pericarditis with hemorrhagic pericardial effusion. Intern Med. 2012; 51: 3197-320I, 2012.

II. Tuon FF, Silva VI, Duboc de Almeida GM, Antonange L, Ho YL. The usefulness of adenosine deaminase in the diagnosis of tuberculous pericarditis. Rev Inst Me Trop S Paulo. 2007;49(3): I65-I70.

12. Florián MC, Franco S, Santacruz D, Montoya KF. Pericarditis tuberculosa: presentación de un caso y revisión de la literatura. Rev Colomb Cardiol. 20I I; I8(5):282-287.

CORRESPONDENCIA: raulhv45@gmail.com

FECHA DE RECEPCIÓN: 29 de diciembre de 2018. FECHA DE ACEPTACIÓN: 30 de marzo de 2019. 